\title{
Fracturas de radio distal. ¿Progresa el desplazamiento entre la segunda y la sexta semana?.
}

\author{
DOI: http//dx.doi.org/10.37315/SOTOCAV20202825561
}

\begin{abstract}
CAPÓ SOLIVERES I, PICAZO GABALDÓN BR, ORENGA MONTOLIU S, GRACIA OCHOA M, ESCRIBANO ZACARÉS S, VILLANUEVA
\end{abstract} DOLCET C.

HOSPITAL FRANCESC DE BORJA DE GANDÍA.

\section{Resumen.}

Las fracturas de radio distal son las más frecuentes en nuestra especialidad. El tratamiento conservador con inmovilización, basado en una serie de parámetros radiológicos, sigue siendo el más utilizado hoy en día. El objetivo de nuestro estudio ha sido evaluar el desplazamiento secundario de estas fracturas entre la segunda y sexta semana de evolución. Para ello hemos evaluado las fracturas de radio distal tratadas de forma conservadora en nuestro Servicio, durante el año 2016. De un total de 53 fracturas de muñeca tratadas de forma conservadora, un $49 \%$ de los casos presentó algún tipo de desplazamiento, si bien la mayor parte de estos tuvieron lugar antes de la segunda semana. Nuestros resultados fueron similares a los revisados en la literatura, en cuanto a datos demográficos y tasa de desplazamiento. Por lo que recomendamos intercalar algunos controles radiográficos semanales en aquellas fracturas que presenten desde el inicio algún parámetro de inestabilidad.

Palabras clave: Fracturas radio distal, tratamiento conservador, resultados radiológicos.

\section{Summary.}

Distal radius fractures are the most frequent in our specialty. Conservative treatment with cast immobilization, based on radiographic criteria, is still the most widely used nowadays. The aim of our study is to evaluate the secondary displacement of these fractures between the second and sixth week of evolution. To this end, we have evaluated the distal radius fractures treated conservatively at our Service, during 2016 . Out of 53 wrist fractures treated conservatively, $49 \%$ of the cases presented some type of displacement, although most of these took place before the second week. Our results were similar to those reviewed in the literature, in terms of demographic data and displacement rate. For this reason, we recommend inserting some weekly radiographic controls in fractures that expose some instability parameters from the beginning.

\section{Correspondencia:}

Irina Capó Soliveres.

Avenida de la Medicina, 6, 46702 Gandia, Valencia. 
CAPÓ SOLIVERES I y COLS. Fracturas de radio distal. ¿Progresa el desplazamiento entre la segunda y la sexta semana?.

\section{Introducción}

Las fracturas de radio distal son las más frecuentes en traumatología ${ }^{1}$. Los criterios para decidir el tipo de tratamiento se basan principalmente en parámetros radiológicos. Si bien no existe un consenso generalizado en cuáles son los parámetros de inestabilidad que se deben tener en cuenta $^{2}$, Lafontaine y cols definieron estos parámetros en una angulación dorsal mayor de 20 grados, presencia de fractura cubital, edad del paciente mayor de 60 años, presencia de conminución dorsal y presencia de un trazo intraarticular, como principales factores de riesgo de desplazamiento ${ }^{3}$. Actualmente los criterios que más comunmente se utilizan son la longitud radial mayor de $7 \mathrm{~mm}$, inclinación volar mayor de $0^{\circ}$, angulación radial de más de $10^{\circ}$ y escalón articular menor de $2 \mathrm{~mm}^{4}$.

A pesar de que el tratamiento conservador de estas fracturas sigue siendo una práctica común, estos parámetros radiológicos se modifican con el colapso progresivo de la fractura tras la manipulación y reducción inicial, es lo que se conoce como desplazamiento secundario $^{5}$. Se puede distinguir un desplazamiento temprano, aquél que se produce en los primeros catorce días, y un desplazamiento tardío si se produce pasados los primeros catorce días ${ }^{2}$. Actualmente no existe un consenso sobre el seguimiento radiológico que debe hacerse en éstos pacientes. Algunos autores recomiendan el seguimiento semanal en las primeras tres semanas y otros realizar un control a la segunda y a la sexta semana ${ }^{2,6-8}$.

El objetivo de este estudio es evaluar si existe desplazamiento secundario entre los controles realizados a la segunda y la sexta semanas postreducción con significación terapéutica.

\section{Material y Métodos}

Hemos realizado un estudio retrospectivo sobre las fracturas de radio distal atendidas en nuestro Hospital en 2016.

Los criterios de exclusión fueron pacientes pediátricos, sin seguimiento completo en nuestro hospital, fractura aislada de estiloides radial, fractura asociada de cúbito distal y tratamiento quirúrgico de entrada o por desplazamiento secundario.

Se han analizado los principales parámetros radiológicos relacionados con el pronóstico funcional: angulación radial, angulación volar, longitud radial y escalón articular. Éstos parámetros se han medido pre y post reducción, a la segunda y a la sexta semana en las radiografías seriadas que se les realizaron a los pacientes. (Fig. 1).

Además, también se han recogido los datos demográficos de sexo, edad y el tipo de inmovilización con el que fueron inmovilizados.

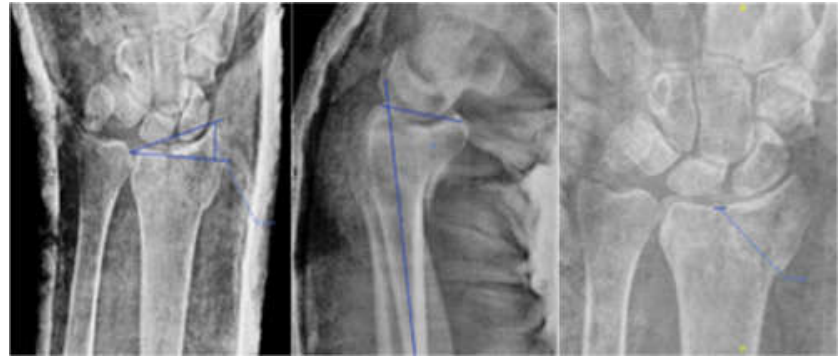

Figura 1. Parámetros radiológicos medidos pre y post reducción, a la segunda y a la sexta semana.

Todos los datos se analizaron con el programa estadístico SPSS Statistics versión 20.

\section{Resultados:}

Tras aplicar los criterios de exclusión de 339 pacientes atendidos en el servicio de Urgencias con el diagnóstico de fractura de radio distal, se excluyeron 90 pacientes pediátricos, 146 pacientes no completaron el seguimiento en nuestro Hospital o los controles radiográficos no se habían realizado dentro del período establecido, 6 pacientes asociaban fractura de cúbito distal, 6 presentaban una fractura aislada de estiloides radial, 35 pacientes recibieron tratamiento quirúrgico en las primeras 2 semanas (29 por presentar indicación quirúrgica desde el primer momento y 6 por desplazamiento secundario entre el control postreducción y el primer control radiográfico), 2 pacientes por presentar un error de diagnóstico.

En total se han estudiado 53 fracturas de radio distal en 52 pacientes, ya que un paciente presentaba fractura bilateral. (Fig. 2).

Atendiendo a los datos demográficos, la edad media de los pacientes era de $68(44-88)$ años. 46 pacientes eran mujeres $(87 \%)$ y 7 varones $(13 \%)$.

La angulación radial media pasó de 17,94 grados a 22,01 postreducción, 19,11 a la segunda semana y 18,6 a la sexta. (Tabla 1).

\begin{tabular}{|l|c|c|c|}
\hline & Media & Mínimo & Máximo \\
\hline Pre-reducción & 17,94 & 8 & 27 \\
\hline Post-reducción & 22,01 & 15 & 28 \\
\hline $2^{\circ}$ semana & 19,11 & 8 & 29 \\
\hline $6^{\circ}$ semana & 18,6 & 11 & 25 \\
\hline
\end{tabular}

Tabla 1. Evolución de la angulación radial en grados. 
CAPÓ SOLIVERES I y COLS. Fracturas de radio distal. ¿Progresa el desplazamiento entre la segunda y la sexta semana?.

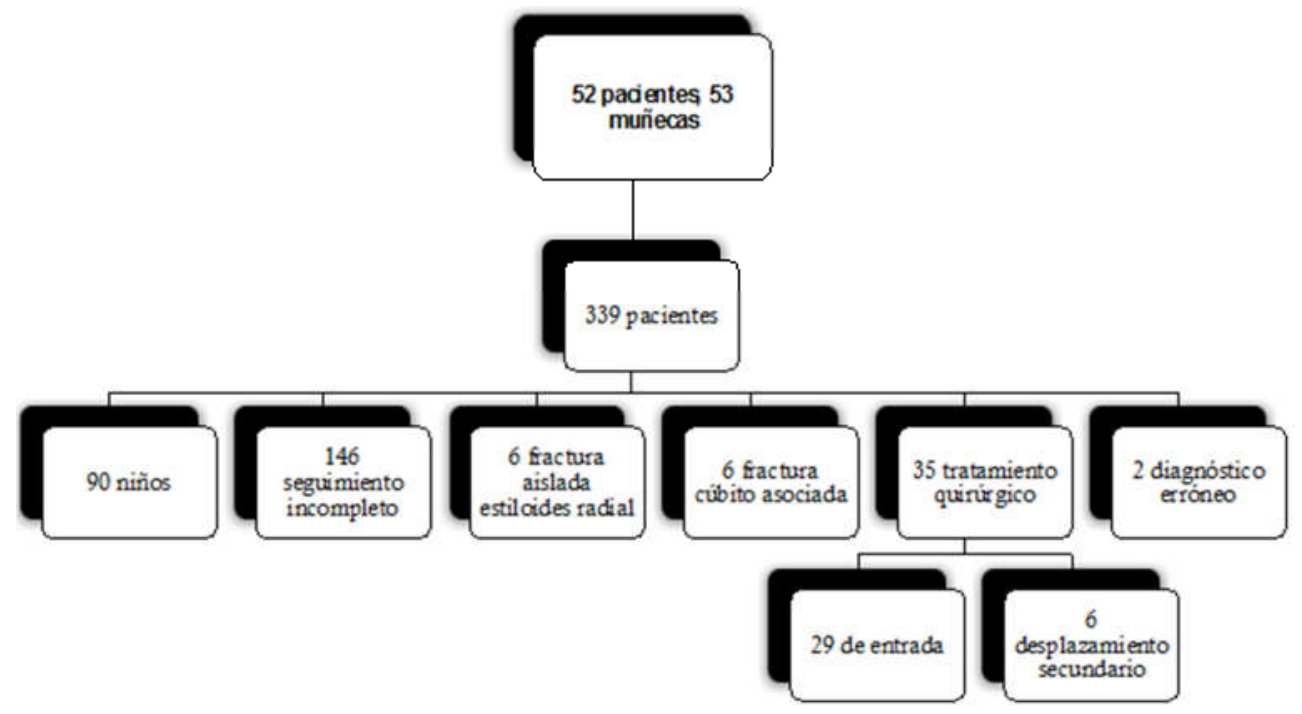

Figura 2. Pacientes estudiados tras aplicar los criterios de exclusión.

La angulación volar media pasó de $-5,94$ grados previo a la reducción a 8,37 postreducción, 2,11 a las dos semanas y 0,15 en la sexta semana. (Tabla 2).

\begin{tabular}{|l|c|c|c|}
\hline & Media & Mi-nimo & Máximo \\
\hline Pre-reducción & $-\mathbf{5 , 9 4}$ & -46 & 40 \\
\hline Post-reducción & $\mathbf{8 , 3 7}$ & -16 & 24 \\
\hline $2^{\text {a semana }}$ & $\mathbf{2 , 1 1}$ & -19 & 22 \\
\hline 6 semana & $\mathbf{0 , 1 5}$ & -24 & 20 \\
\hline
\end{tabular}

Tabla 2. Evolución de la angulación volar en grados. milímetros.

\begin{tabular}{|l|c|c|c|}
\hline & Media & Mínimo & Máximo \\
\hline Pre-reducción & 7,6 & 3 & 13 \\
\hline Post-reducción & 9,45 & 6 & 12,6 \\
\hline $2^{\circ}$ semana & 8,76 & 3 & 15 \\
\hline $6^{\circ}$ semana & 9,5 & 0 & 15 \\
\hline
\end{tabular}

Tabla 3. Evolución de la longitud radial medida en

La longitud radial media previa a la reducción de 7,6mm había pasado a 9,45mm postreducción, $8,76 \mathrm{~mm}$ a las dos semanas y $9,5 \mathrm{~mm}$ a las seis. (Tabla 3 ).

\begin{tabular}{|l|c|}
\hline & Media \\
\hline Pre-reducción & 2,35 \\
\hline Post-reducción & 2,1 \\
\hline $2^{\text {a }}$ semana & 4,5 \\
\hline $6^{\circ}$ semana & 4,2 \\
\hline
\end{tabular}

Tabla 4. Escalón articular medido en milímetros

En cuanto al escalón articular, únicamente dos pacientes lo presentaron, con una media de $2,35 \mathrm{~mm}$ pre y $2,1 \mathrm{~mm}$ postreducción, $4,5 \mathrm{~mm}$ a la segunda semana y 4,2 a la sexta. (Tabla 4). 

semana?.

En cuanto al tipo de inmovilización 41 fracturas fueron inmovilizadas con férula antebraquial dorsal, 6 con yeso antebraquial completo y 6 con férula braquial. Se ha visto que la inmovilización con yeso cerrado tiende a mantener mejor la angulación volar y que la férula antebraquial mantiene mejor la angulación y longitud radial. (Fig. 3).
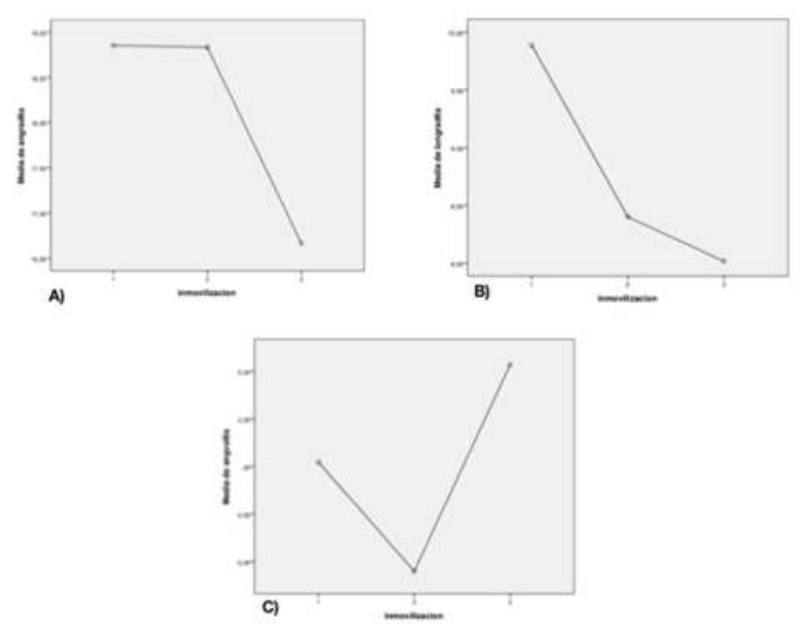

Figura 3: Evolución de las angulaciones y longitud según el tipo de inmovilización. A) Angulación radial. B) Longitud radial. C) Angulación volar. 1: férula antebraquial. 2: férula braquial. 3: yeso antebraquial completo.

Atendiendo exclusivamente a los criterios radiológicos de indicación quirúrgica más aceptados de mínimo $7 \mathrm{~mm}$ de longitud radial, $0^{\circ}$ de inclinación volar, angulación radial de más de $10^{\circ}$ y escalón articular menor de $2 \mathrm{~mm}$, hemos obtenido un total de 26 casos de desplazamiento secundario $(49 \%)$.

De éstos, en catorce casos $(26,41 \%)$ el desplazamiento tuvo lugar entre la radiografía de control postreducción y el control a la segunda semana, es decir, un desplazamiento temprano. De los cuales, tres casos tuvieron un desplazamiento muy significativo, que sobrepasaba los límites tolerables de deformidad. En estos casos aunque habría estado indicado el tratamiento quirúrgico, se desestimó por ciertas características de los pacientes, como comorbilidades, riesgo quirúrgico, baja demanda funcional, etc.

Los otros doce casos $(22,6 \%)$ en los que existe un importante desplazamiento que podrían haber supuesto un cambio de indicación del tratamiento conservador al quirúrgico, el desplazamiento fue entre la segunda y sexta semana (Fig. 4). De éstos, sólo un caso sobrepasaba los límites tolerables de deformidad.

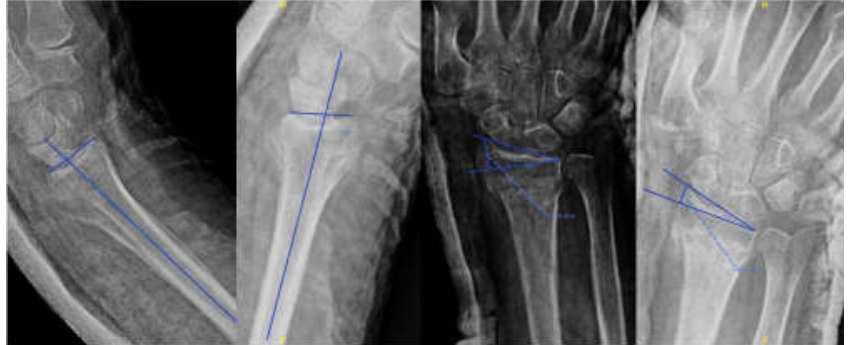

Figura 4. Ejemplo de desplazamiento entre la segunda y sexta semana.

\section{Discusión}

Nuestros resultados son comparables a otros estudios en cuanto a la distribución por sexo y edad media de los pacientes ${ }^{4-6}$. Una distribución tan desigual por sexos se debe a que este tipo de fracturas son más frecuentes en las mujeres, sobre todo con impactos de baja energía. En los hombres los traumatismos son de alta energía, por lo que las fracturas son más inestables ${ }^{5}$ y la mayoría de los varones de nuestra serie fueron tratados mediante cirugía por cumplir criterios de inestabilidad ya en el momento del diagnóstico.

En cuanto a la pérdida de reducción, nuestra tasa de pérdida de reducción fue del $49 \%$ en total, diferenciándola en un $26 \%$ temprana y un $22 \%$ tardía, es comparable o incluso algo menor a la identificada en la literatura.

Myderrizi obtuvo una pérdida de reducción global en el 52\% de los casos ${ }^{4}$. Jung y cols. obtuvieron una tasa de pérdida de reducción del $25 \%$ en las dos primeras semanas, y del $11 \%$ tardía $^{9}$. En el grupo de Neidenbach y cols. la pérdida de reducción fue más obvia en las primeras seis semanas ${ }^{6}$. En el estudio de Tahririan y cols. la tasa de desplazamiento secundario es del $58.6 \%$, siendo éste principalmente en las dos primeras semanas tras reducción ${ }^{5}$.

No existe consenso en la literatura en cuanto a los límites tolerables de deformidad ${ }^{10}$, aunque Fourrier describió los límites de los parámetros radiográficos más allá de los cuales aumenta progresivamente el porcentaje de malos resultados funcionales. Éstos son: angulación radial entre $20^{\circ}$ y $30^{\circ}$, angulación volar entre $10^{\circ}$ y $20^{\circ}$, índice radiocubital entre 0 y $-2 \mathrm{~mm}^{11}$. Sin embargo, según Fernández, se puede aceptar cierta deformidad individualizando cada caso según las demandas funcionales del paciente ${ }^{8}$. Otros autores como Synn y cols. defienden que los resultados funcionales son independientes del resultado radiológico final ${ }^{10}$.

Con nuestro estudio hemos objetivado que el desplazamiento a la segunda semana no es definitivo, sino que hasta en un $22 \%$ de los casos la deformidad puede continuar progresando. Sin embargo, hasta la fecha ninguno de estos pacientes ha precisado una cirugía de secuelas. 
CAPÓ SOLIVERES I y COLS. Fracturas de radio distal. ¿Progresa el desplazamiento entre la segunda y la sexta semana?.

Una de las principales limitaciones de nuestro estudio es que los resultados se basan únicamente en el análisis radiológico y no hemos tenido en cuenta los resultados funcionales. Sabemos que tenemos algunos casos en los que en la radiografía final algunos parámetros radiológicos no son aceptables, pero no tienen una significación en la funcionalidad, especialmente en pacientes de mayor edad, con baja demanda funcional.

\section{Conclusiones}

Clásicamente el manejo de las fractura de radio distal ha sido conservador en aquellas fracturas que se consideran estables. Si bien se ha visto que incluso las fracturas que parecen muy estables tras la reducción inicial tienden a colapsarse.

Ante la progresión radiológica del desplazamiento entre la segunda y la sexta semana que hemos apreciado en nuestro trabajo, nosotros consideramos adecuado intercalar nuevos controles radiográficos en pacientes susceptibles de tratamiento quirúrgico para evitar casos con angulaciones y deformidades no deseables, especialmente en aquellos pacientes en los que en el control de la segunda semana ya presentan algún criterio de inestabilidad.

Recomendamos aumentar el seguimiento, para asegurarnos un control más exhaustivo que nos permita modificar la indicación a un tratamiento quirúrgico cuando el desplazamiento aun no sea muy severo.

En nuestro estudio nos hemos centrado en la evolución radiológica de estas fracturas ya que creemos que es una de nuestras principales herramientas a la hora de tomar una decisión terapéutica. Si bien se tiene en cuenta en cada caso las características funcionales de los pacientes, como pueden ser comorbilidades, riesgo quirúrgico, demanda funcional, edad, mano dominante etc.

Aunque no siempre se pueden relacionar los resultados radiológicos con los resultados funcionales, quedaría pendiente confirmar cómo han influido esos desplazamientos en el resultado funcional de los pacientes, si bien sabemos que algunas deformidades son muy bien toleradas por los mismos.

\section{Bibliografía}

1. Einsiedel T, Freund W, Sander S, Trnavac S, Gebhard F, Kramer M. Can the displacement of a conservatively treated distal radius fracture be predicted at the beginning of the treatment? Int Orthop 2009; 33: 795-800.

2. Makhni EC, Taghinia A, Ewald T, Zurakowski D. Conminution of the dorsal metaphysis and its effects on the radiographic outcomes of distal radius fractures. J Hand Surg Eur Vol 2010 Oct; 35E(8): 652-58.

3. Lafontaine M, Hardy D, Deline P. Stability assessment of distal radius fractures. Injury. 1989; 20:208-10.

4. Myderrizi N. Factors predicting late Collapse of Distal Radius Fractures. Malays Orthop J. 2011; 5(3):3-7.

5. Tahririan MA, Javdan M, Nouraei MH, Dehghani M. Evaluation of instability factors in distal radius fractures. J Res Med Sci 2013 Oct; 18(10):892-96.

6. Neidenbach P, Audigé L, Wilhelmi-Mock M, Hanson B, De Boer P. The efficacy of closed reduction in displaced distal radius fractures. Injury 2010; 41:592-98.

7. Földhazy Z, Tórnkvist H, Elmstedt E, Andersson G, Hagsten B, Ahrengart L. Long-term Outcome of Nonsurgically treated Distal Radius Fractures. J Hand Surg Am. 2007 Nov; 9(32A):1354-84.

8. Fernández DL. Closed manipulation and casting of distal radius fractures. Hand Clin 2005; 21:307-16.

9. Jung $\mathrm{H}$, Hong H, Jung HJ, Kim JS, Park HY, Bae KH, Jeon I. Redisplacement of Distal Radius Fracture after initial Closed Reduction: analysis of prognostic factors. Clin Orthop Surg. 2015; 7:377-82.

10. Synn AJ, Makhni EC, Makhni MC, Rozental TD, Day CS. Distal Radius Fractures in older patients. Clin Orthop Relat Res. 2009; 467:1612-20.

11. Fourrier P, Bardy A, Roche G, Cisterne JP, Chambon A. Approche d'une définition du cal vicieux du poignet. Int Orthop 198; 4:299-305. 